\title{
An Enhanced Iterative Flipping PTS Technique for PAPR Reduction of OFDM Signals
}

\author{
Byung Moo Lee ${ }^{1}$ and Rui J. P. de Figueiredo ${ }^{2}$ \\ ${ }^{1}$ Central RED Laboratory, Korea Telecom (KT), \\ Seoul, 137-792, Korea, \\ Email:blee@kt.com \\ ${ }^{2}$ Laboratory for Intelligent Signal Processing and Communications, \\ Department of Electrical Engineering and Computer Science, \\ University of California, Irvine, CA 92697-2625, USA,
} Email:rui@uci.edu

\section{Introduction}

Orthogonal Frequency Division Multiplexing (OFDM) has several desirable attributes, such as high immunity to inter-symbol interference, robustness with respect to multi-path fading, and ability for high data rates, all of which are making OFDM to be incorporated in wireless standards like IEEE 802.11a/g/n WLAN and ETSI terrestrial broadcasting. However one of the major problems posed by OFDM is its high Peak-to-Average-Power Ratio (PAPR), which seriously limits the power efficiency of the transmitter's High Power Amplifier (HPA). This is because PAPR forces the HPA to operate beyond its linear range with a consequent nonlinear distortion in the transmitted signal.

One of good solutions to mitigate this nonlinear distortion is put a Pre-Distorter before the High Power Amplifier and increase linear dynamic range up to a saturation region (1) (2) (3). However, the main disadvantage of Pre-Distorter technique is that these PD techniques only work in a limited range, that is, up to the saturation region of the amplifier. In this situation, Peak-to-Average Power Ratio (PAPR) reduction techniques which pull down high PAPR of OFDM signal to an acceptable range can be a good complementary solution. Due to practical importance of this, there are various PAPR reduction techniques for OFDM signals (4) (5) (6) (7) (8) (9). Among them, the PTS (Partial Transmit Sequence) technique is very promising because it does not give rise to any signal distortion (9). However, its high complexity makes it difficult to use in a practical system. To solve the complexity problem of the PTS technique, Cimini and Sollenberger proposed an iterative flipping algorithm (10). Even though the iterative flipping algorithm greatly reduces the complexity of the PTS technique, there is still some performance gap between the ordinary PTS and the iterative flipping algorithm.

In this chapter, we propose an enhanced version of the iterative flipping algorithm to reduce the performance gap between the iterative flipping algorithm and the ordinary PTS technique. In the proposed algorithm, there is an adjustable parameter to allow a performance/complexity trade-off. 


\section{OFDM and Peak-to-Average Power Ratio (PAPR)}

An OFDM signal of $N$ subcarriers can be represented as

$$
x(t)=\frac{1}{\sqrt{N}} \sum_{k=0}^{N-1} X[k] e^{j 2 \pi f_{k} t}, 0 \leq t \leq T_{S}
$$

where $T_{s}$ is the duration of the OFDM signal and $f_{k}=\frac{k}{T_{s}}$.

The high PAPR of the OFDM signal arises from the summation in the above IDFT expression. The PAPR of the OFDM signal in the analog domain can be represented as

$$
P A P R_{c}=\frac{\max _{0 \leq t \leq T_{s}}|x(t)|^{2}}{E\left(|x(t)|^{2}\right)}
$$

Nonlinear distortion in HPA occurs in the analog domain, but most of the signal processing for PAPR reduction is performed in the digital domain. The PAPR of digital domain is not necessarily the same as the PAPR in the analog domain. However, in some literature (11) (12) (13), it is shown that one can closely approximate the PAPR in the analog domain by oversampling the signal in the digital domain. Usually, an oversampling factor $L=4$ is sufficient to satisfactorily approximate the PAPR in the analog domain. For these reasons, we express PAPR of the OFDM signal as follows.

$$
P A P R=\frac{\max _{0 \leq n \leq L N}|x(n)|^{2}}{E\left(|x(n)|^{2}\right)}
$$

\section{Existing PTS Techniques}

The PTS technique is a powerful PAPR reduction technique first proposed by Muller and Huber in (9). Thereafter various related papers have been published. In this section, we show two representative PTS techniques, the original PTS technique and Cimini and Sollenberger's iterative flipping technique (10).

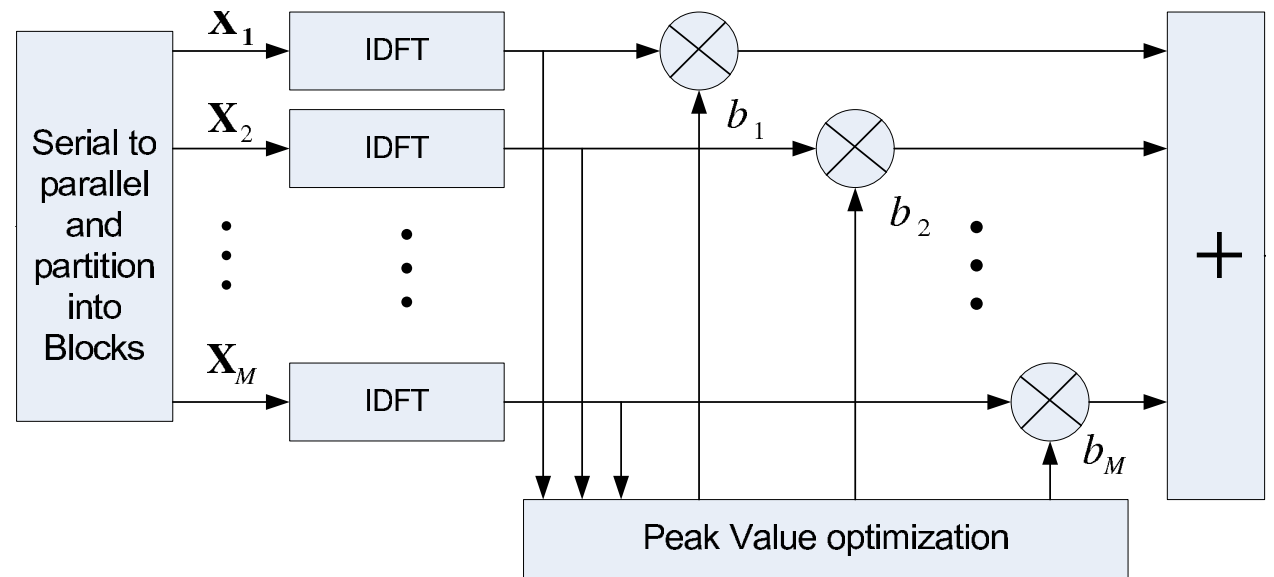

Fig. 1. Block diagram of the PTS scheme 


\subsection{Ordinary PTS Technique}

A block diagram of the PTS technique is shown in Figure 1. The algorithm of the original PTS technique can be explained as follows.

First, the signal vector is partitioned into $M$ disjoint subblocks which can be represented as

$$
\mathbf{X}_{\mathbf{m}}=\left[X_{m, 0}, X_{m, 1}, \cdots, X_{m, N-1}\right]^{T}, m=1,2, \cdots, M
$$

All the subcarrier positions which are presented in other subblocks must be zero so that the sum of all the subblocks constitutes the original signal, i.e,

$$
\sum_{m=1}^{M} \mathbf{X}_{\mathbf{m}}=\mathbf{X}
$$

Each subblock is converted through IDFT into an OFDM signal $\mathbf{x}_{\mathbf{m}}$ with oversampling, which can be represented as

$$
\mathbf{x}_{\mathbf{m}}=\left[x_{m, 0}, x_{m, 1}, \cdots, x_{m, N L-1}\right]^{T}, m=1,2, \cdots, M
$$

where $L$ is the oversampling factor. After that, each subblock is multiplied by a different phase factor $b_{m}$ to reduce PAPR of the OFDM signal. The phase set can be represented as

$$
P=\left\{e^{j 2 \pi w / W} \mid w=0,1, \cdots, W-1\right\}
$$

where $W$ is the number of phases.

Because of the high computational complexity of the PTS technique, one generally uses only a few phase factors. The choice, $b_{m} \in\{ \pm 1, \pm j\}$, is very interesting since actually no multiplication is performed to rotate the phase (14). The peak value optimization block in Figure 1 iteratively searches the optimal phase sequence which shows minimum PAPR. Finding optimal PAPR using PTS PAPR reduction technique can be represented as

$$
P A P R_{\text {optimal }}=\frac{\min _{b_{1}, \cdots b_{M}}\left(\max _{0 \leq n \leq L N}\left|\sum_{m=1}^{M} b_{m} x_{m, n}\right|^{2}\right)}{E\left(|x(n)|^{2}\right)}
$$

This process usually requires large computational power. After finding the optimal phase sequence which minimizes PAPR of the OFDM signal, all the subblocks are summed as in the last block of Figure 1 with multiplication of the optimal phase sequence. Then the transmit sequence can be represented as

$$
\begin{aligned}
\mathbf{x}^{\prime}(\mathbf{b}) & =\left[\mathbf{x}_{\mathbf{1}}, \mathbf{x}_{\mathbf{2}}, \cdots, \mathbf{x}_{\mathbf{M}}\right]\left[\begin{array}{c}
b_{1} \\
b_{2} \\
\vdots \\
b_{M}
\end{array}\right] \\
& =\sum_{m=1}^{M} b_{m} \cdot \mathbf{x}_{\mathbf{m}}
\end{aligned}
$$

Here we assume $\mathbf{b}^{\mathbf{T}}=\left[\begin{array}{llll}b_{1} & b_{2} & \cdots & b_{M}\end{array}\right]$ is an optimal phase set which gives minimum PAPR among various phase sets. 


\subsection{Iterative Flipping PTS Technique}

Cimini and Sollenberger's iterative flipping technique is developed as a sub-optimal technique for the PTS algorithm. In their original paper (10), they only use binary weighting factors. That is $b_{m}=1$ or $b_{m}=-1$. These can be expanded to more phase factors. The algorithm is as follows. After dividing the data block into $M$ disjoint subblocks, one assumes that $b_{m}=1,(m=1,2, \cdots, M)$ for all of subblocks and calculates PAPR of the OFDM signal. Then one changes the sign of the first subblock phase factor from 1 to $-1\left(b_{1}=-1\right)$, and calculates the PAPR of the signal again. If the PAPR of the previously calculated signal is larger than that of the current signal, keep $b_{1}=-1$. Otherwise, revert to the previous phase factor, $b_{1}=1$. Suppose one chooses $b_{1}=-1$. Then the first phase factor is decided, and thus kept fixed for the remaining part of the algorithm. Next, we follow the same procedure for the second subblock. Since one assumed all of the phase factors were 1 , in the second subblock, one also changes $b_{2}=1$ to $b_{2}=-1$, and calculates the PAPR of the OFDM signal. If the PAPR of the previously calculated signal is larger than that of the current signal, keep $b_{2}=-1$. Otherwise, revert to the previous phase factor, $b_{2}=1$. This means the procedure with the second subblock is the same as that with the first subblock. One continues performing this procedure iteratively until one reaches the end of subblocks $\left(M^{\text {th }}\right.$ subblock and phase factor $\left.b_{M}\right)$. A similar technique was also proposed by Jayalath and Tellambura (16). The difference between the Jayalath and Tellambura's technique and that of Cimini and Sollenberger is that, in the former, the flipping procedure does not necessarily go to the end of subblocks ( $M^{\text {th }}$ block). To reduce computational complexity, the flipping is stopped before the end of the entire procedure if the desired PAPR OFDM signal achieved at that point.

\section{Enhanced Iterative Flipping PTS Technique}

In this section, we present an Enhanced Iterative Flipping PTS (defined by EIF-PTS) technique which is a modified version of the Cimini and Sollenberger's Iterative Flipping PTS (IF-PTS) technique. We use, in this chapter, 4 phase factors to reduce the PAPR of the OFDM signal, that is, $W=4\left(b_{m} \in\{ \pm 1, \pm j\}\right)$.

As explained earlier, in the iterative flipping algorithm, one keeps only one phase set in each subblock. Even though the phase set chosen in the first subblock shows minimum in the first subblock, that is not necessarily minimum if we allow it to change until we continue the procedure up to the end subblock. The basic idea of our proposed algorithm is that we keep more phase factors in the first subblock rather than keep only one phase factor, and delay the final decision to the end of subblock. We can choose the number of phase factors that we will keep by adjusting a parameter, $S$ where $S$ is the number of phase factors which we will keep in the first subblock. The larger $S$, the better performance we get but with higher complexity. The basic structure of the Enhanced Iterative Flipping Partial Transmit Sequence (EIF-PTS) is illustrated in Figure 2, for the case in which $S=W=4$. In this illustration, each of four phases $b_{11}=1, b_{12}=-1, b_{13}=j, b_{14}=-j$ is multiplied successively by the first subblock of the signal thus generating four phase sequences, $S_{1}, S_{2}, S_{3}$ and $S_{4}$. Then for each $S_{i}$, from the second subblock, the IF (Iterative Flipping) algorithm of Cimini and Sollenberger is performed. At the end of application of this procedure up to the end subblock for respectively $S_{1}, S_{2}, S_{3}$ and $S_{4}$, there will be four sequences $\tilde{S}_{1}, \tilde{S}_{2}, \tilde{S}_{3}$ and $\tilde{S}_{4}$, each having respectively $b_{1 i}$ for the first sbublock of $\tilde{S}_{i}$, and different phases generated by the application of the IF procedure to each of the four sequences. At the conclusion of this procedure, the EIF-PTS algorithm chooses the $\tilde{S}_{i}, i=1,2,3,4$ which gives rise to the lowest PAPR. For the clarity, we provide an example in Table 1, Table 2 and Table 3. 


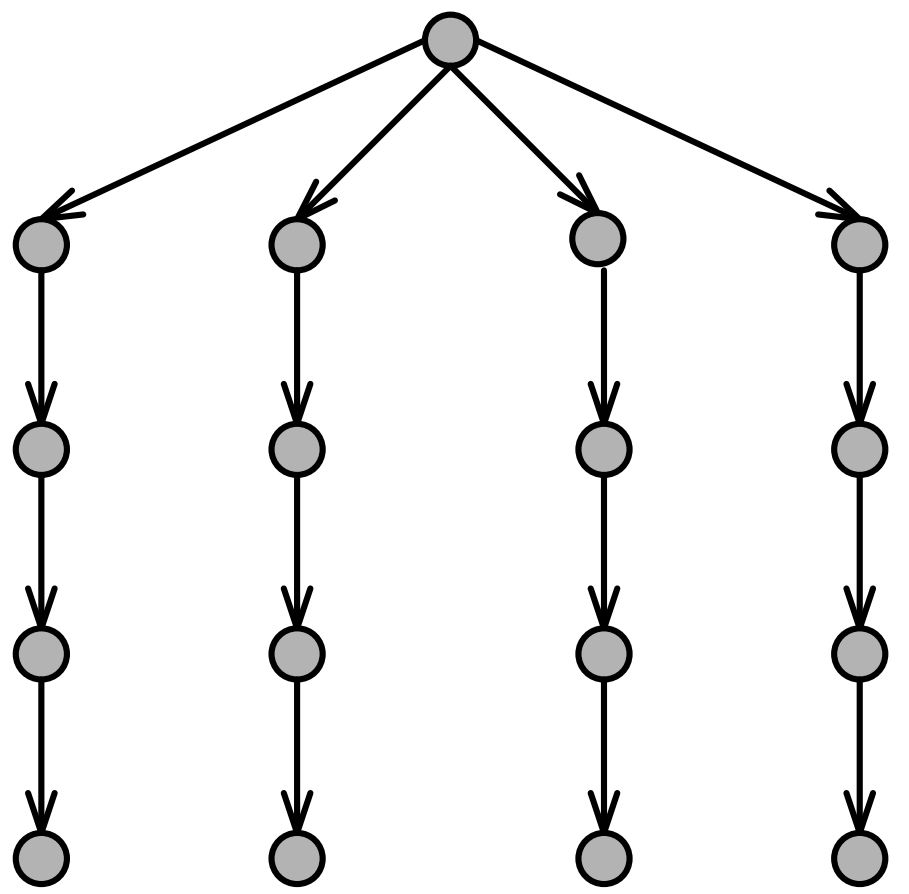

Fig. 2. Structure of an Enhanced iterative flipping algorithm $(S=4)$

In summary, we perform following procedure to efficiently improve the iterative flipping algorithm.

1. Choose the parameter, $S$ to decide how many phase factors we will keep in the first subblock depending on the performance/complexity, where $1 \leq S \leq W$.

2. Keep the $S$ phase sequences which show minimum PAPRs in the first subblock.

3. From each node which was kept in the first subblock, do iterative flipping algorithm until you reach the end of subblock.

4. At the end of subblock, find the phase sequence and signal which show minimum PAPR and choose it as a final decision.

It is also worth noting that when $S=1$, the proposed algorithm is equivalent to the iterative flipping algorithm.

\section{Simulation Results and Discussion}

In this section, we show simulation results of the proposed EIF (Enhanced Iterative Flipping) PTS algorithm. We use 16QAM OFDM with $N=64$ subcarriers. We divide the one signal block as $M=4$ adjacent/disjoint subblocks and use $W=4\left(b_{m} \in\{ \pm 1, \pm j\}\right)$ phase factors. We oversampled the data by $L=4$ to estimate PAPR of the continuous time signal. The first simulation result is shown in Figure 3. In this figure, the $x$-axis denotes PAPR value in dB scale while the $y$-axis, the respective Complementary Cumulative Distribution Function (CCDF) or 


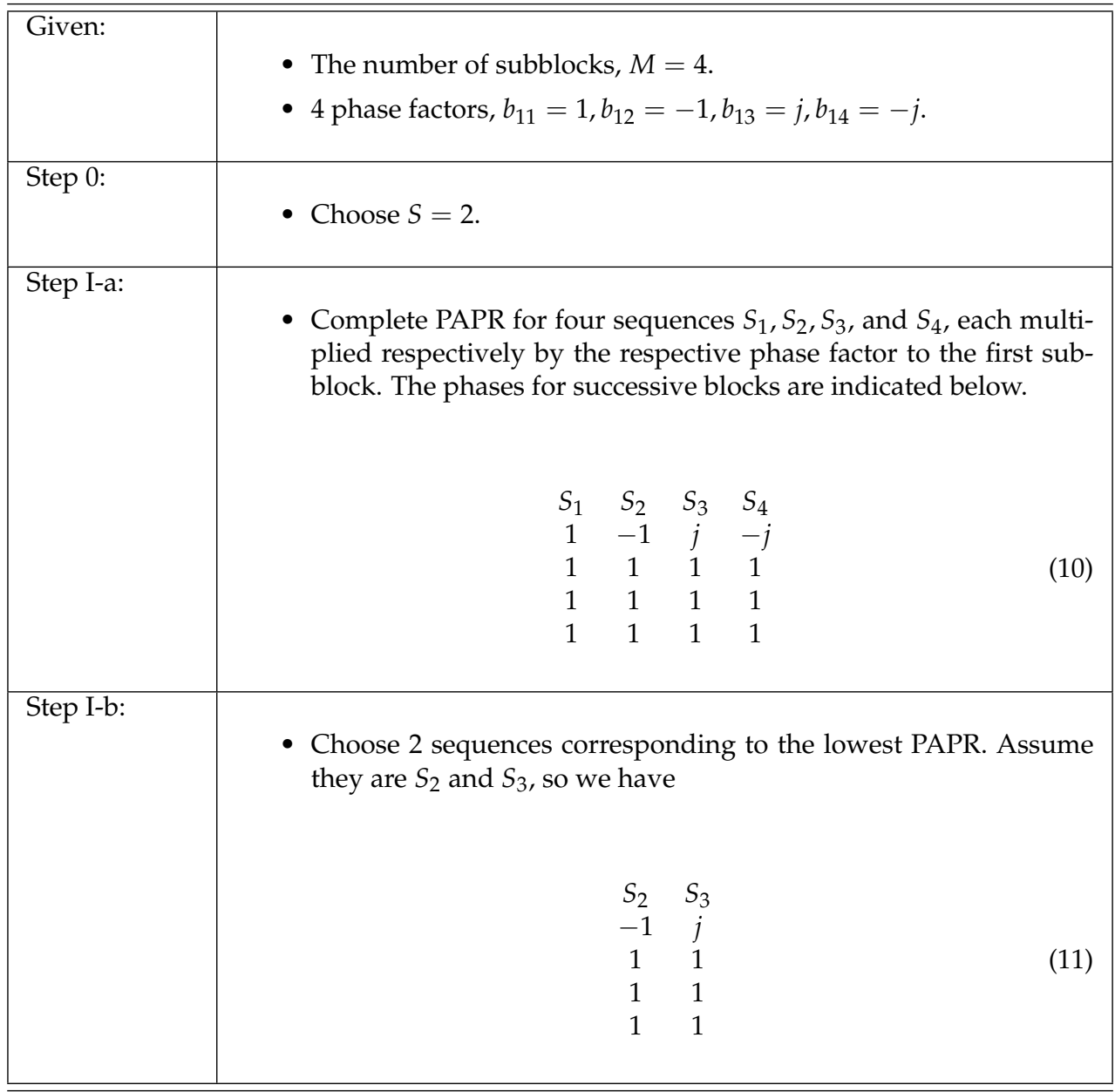

Table 1. Example of EIF-PTS technique $(S=2)(1)$

clipping probability. As we can see in Figure 3, the proposed algorithm reduces the PAPR of the OFDM signal by more than $2 \mathrm{~dB}$ at the $0.1 \%$ of CCDF. The performance degradation between the EIF-PTS and ordinary PTS is only less than $0.5 \mathrm{~dB}$. The complexity of ordinary PTS can be represented as

$$
\text { The number of iterations of ordinary PTS }=W^{(M-1)}
$$

In this chapter, we assume the complexity is only dependent on the number of iterations. The reason, for the number of iterations of ordinary PTS is $W^{M-1}$, and not $W^{M}$ is that ordinary PTS can fix the phase factor of the first subblock without any performance penalty. The complexity 


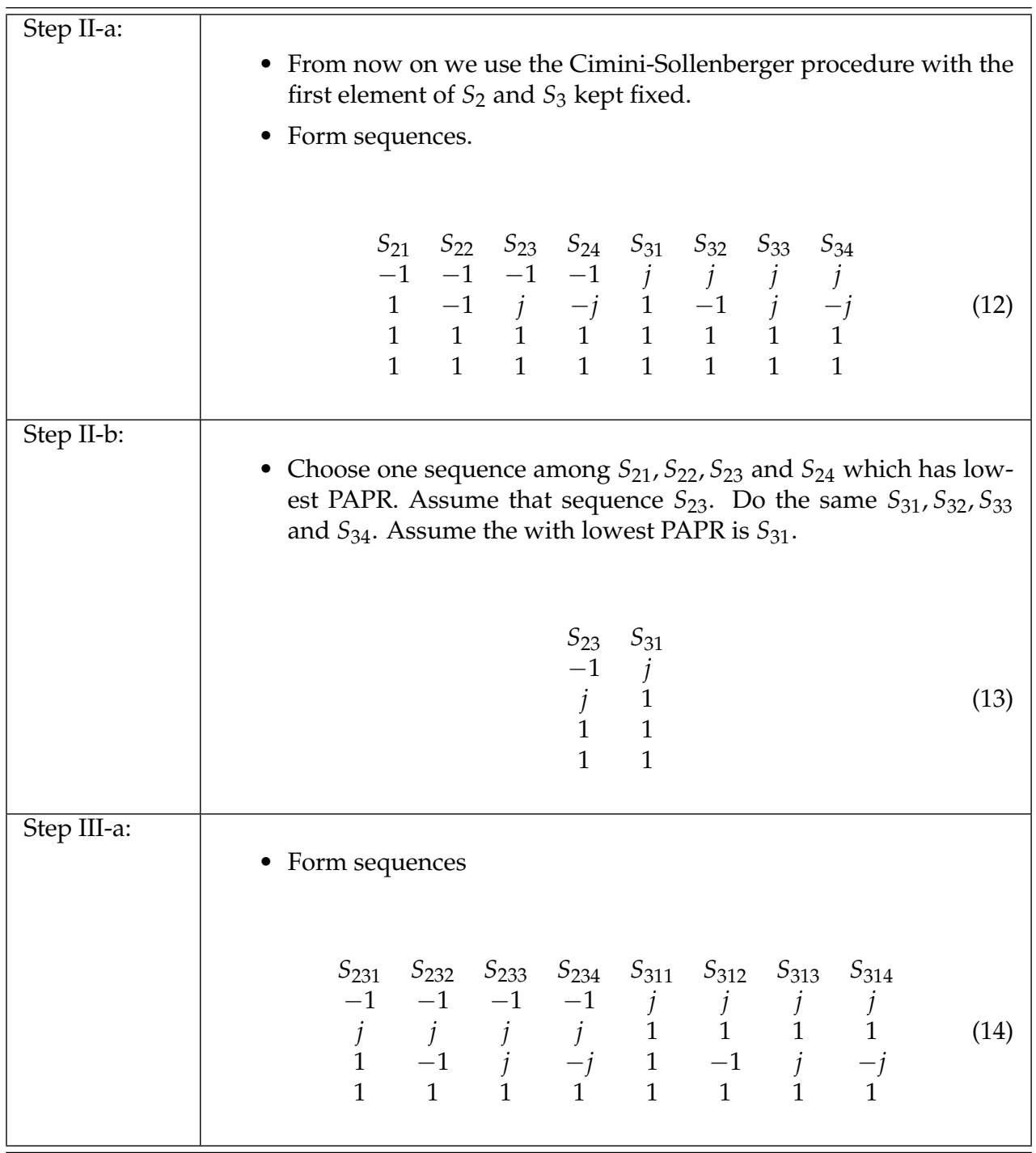

Table 2. Example of EIF-PTS technique $(S=2)(2)$

of the proposed EIF-PTS can be represented as

The Number of Iterations of Proposed Algorithm $=$ $W+(W-1) \cdot(M-1) \cdot S$

We organize complexities of the proposed Enhanced Iterative Flipping (EIF) PTS and ordinary PTS in Table 4. The proposed EIF-PTS algorithm also can fix the first subblock (F-EIF-PTS). 


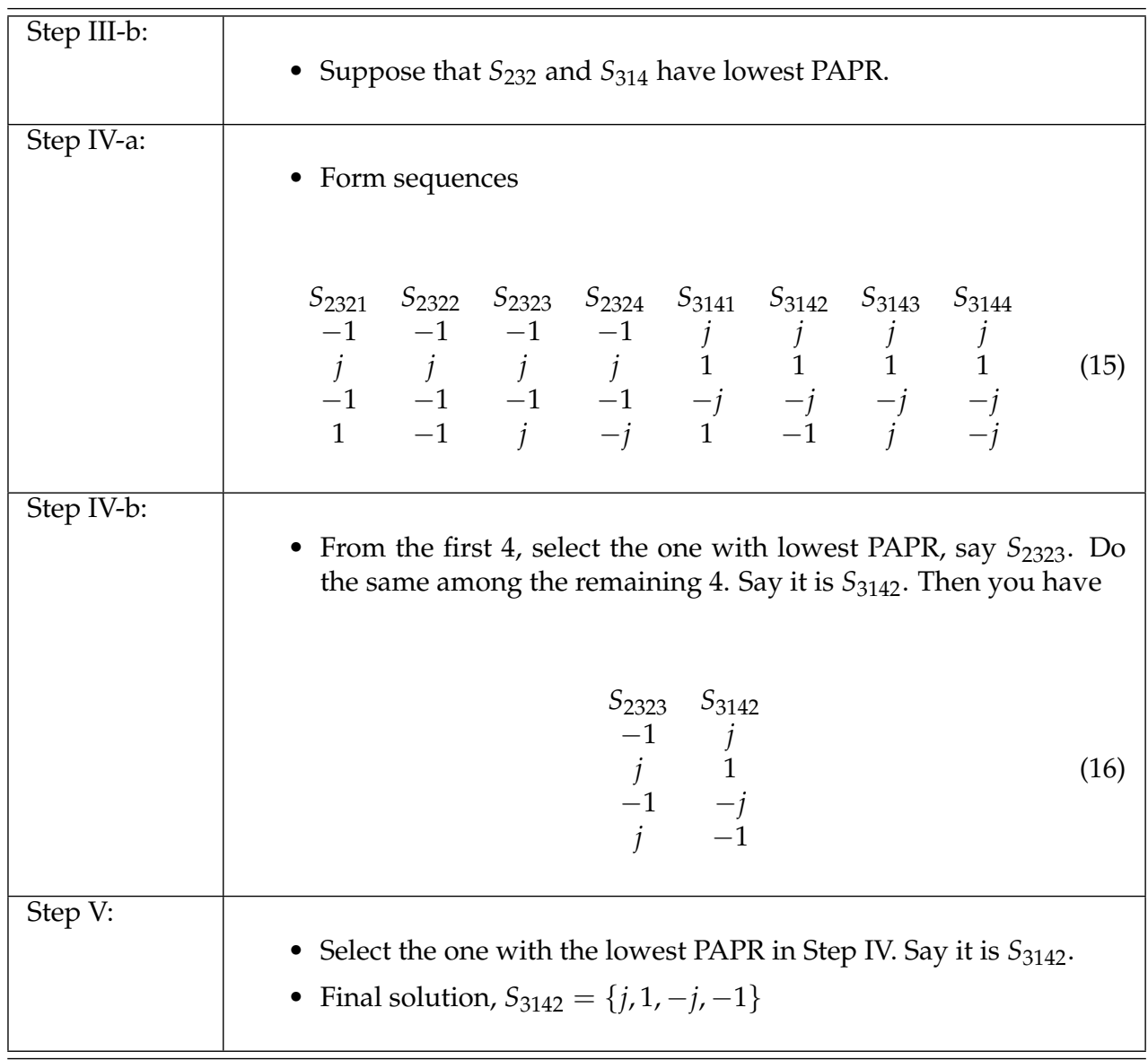

Table 3. Example of EIF-PTS technique $(S=2)(3)$

\begin{tabular}{||c||c||}
\hline \multicolumn{1}{||c||}{} & Number of iterations \\
\hline \hline EIF-PTS, $S=1$ & 13 \\
\hline EIF-PTS, $S=2$ & 22 \\
\hline EIF-PTS, $S=3$ & 31 \\
\hline EIF-PTS, $S=4$ & 40 \\
\hline Ordinary PTS & 64 \\
\hline
\end{tabular}

Table 4. Comparison of complexities between EIF-PTS and Ordinary PTS

However, in this case, we get some performance penalty. The simulation results and comparison of complexity of this case is in Figure 4 and Table 5. It is obvious that, in this case, we can 


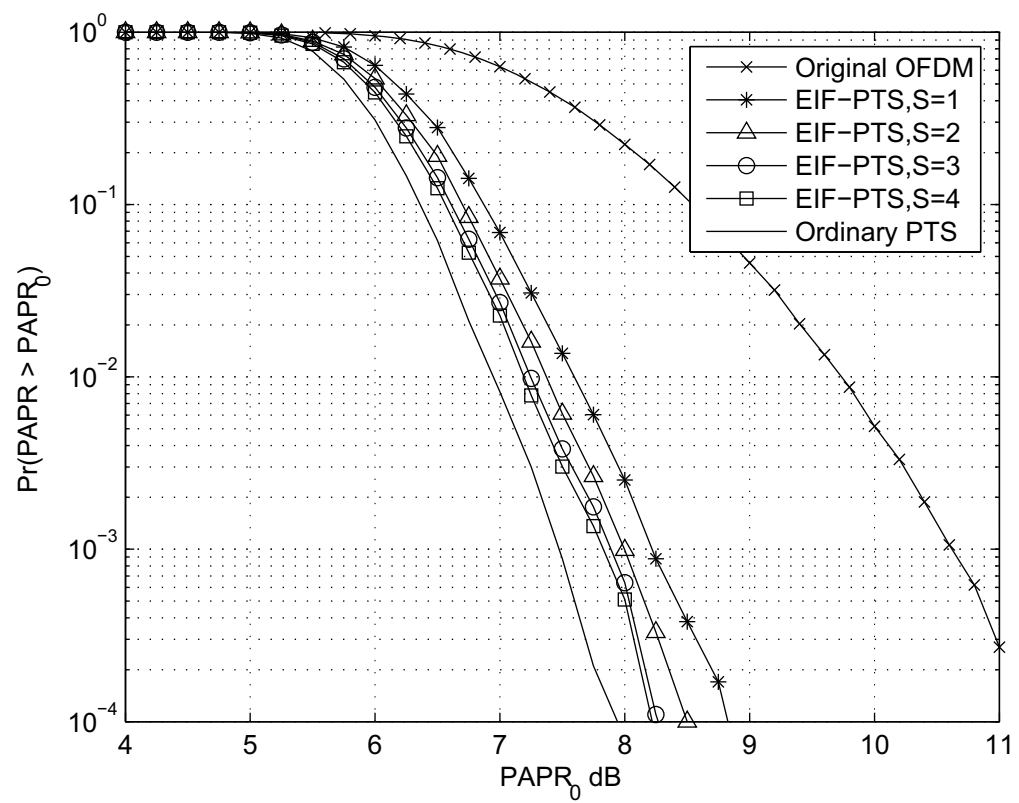

Fig. 3. Performance of EIF-PTS, $M=4$

represent the number of iterations as follows.

The Number of Iterations of Proposed Algorithm $=$

$$
W+(W-1) \cdot(M-2) \cdot S
$$

This fixed technique, which we call F-EIF-PTS, is needed if we try to send SI (Side Information) to the receiver. To embed SI, at least one block of phase should not be changed.

\begin{tabular}{||c||c||}
\hline \multicolumn{1}{||c||}{} & Number of iterations \\
\hline \hline EIF-PTS, $S=1$ & 10 \\
\hline EIF-PTS, $S=2$ & 16 \\
\hline EIF-PTS, $S=3$ & 22 \\
\hline EIF-PTS, $S=4$ & 28 \\
\hline Ordinary PTS & 64 \\
\hline
\end{tabular}

Table 5. Comparison of complexities between EIF-PTS and Ordinary PTS, when the first phase factor is fixed as 1

Now we increase the number of subblocks from $M=4$ to $M=8$. In Figure 5, as an ordinary PTS approximation, we refer (15).

The performance gap is larger than that of the previous case $(M=4)$. However, the complexity gap is much larger than the performance gap. The comparison of the complexity is provided in Table 6 . The performance difference between EIF-PTS, $S=4$ and ordinary PTS is less 


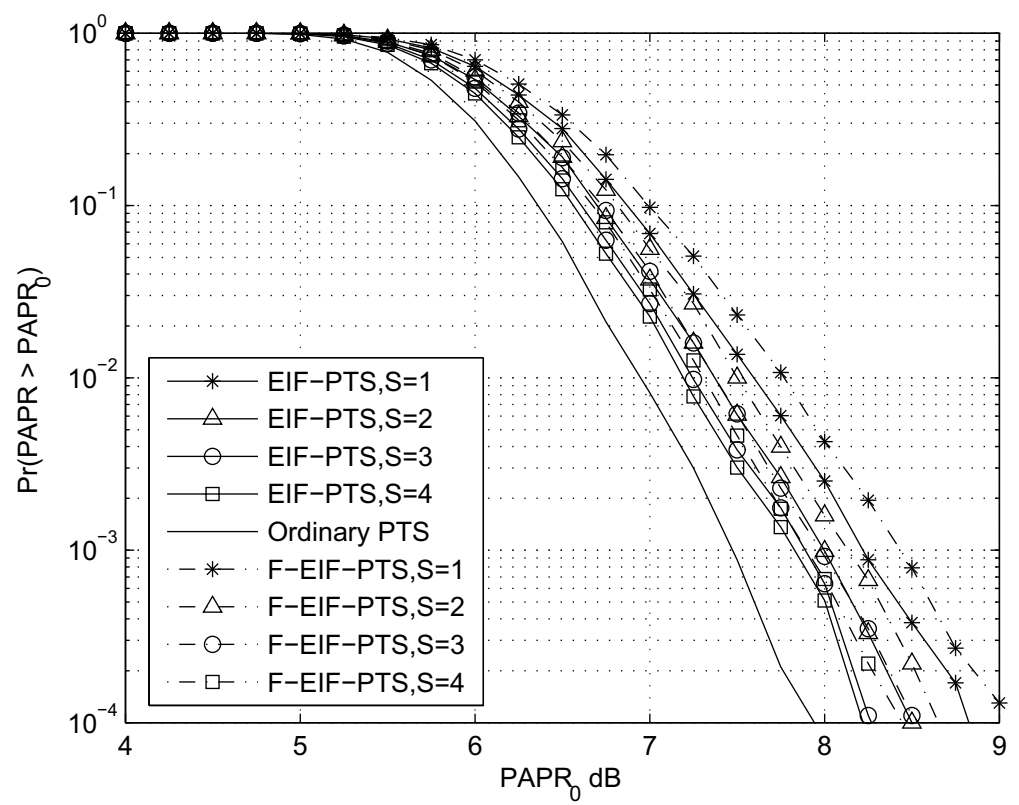

Fig. 4. Performance of EIF-PTS, when fixed the first phase factor (F-EIF-PTS) and normal EIF-PTS case

\begin{tabular}{||c||c||}
\hline \multicolumn{1}{||c||}{} & Number of iterations \\
\hline \hline EIF-PTS, $S=1$ & 25 \\
\hline EIF-PTS, $S=2$ & 46 \\
\hline EIF-PTS, $S=3$ & 67 \\
\hline EIF-PTS, $S=4$ & 88 \\
\hline Ordinary PTS & 16384 \\
\hline \hline
\end{tabular}

Table 6. Comparison of complexities between EIF-PTS and Ordinary PTS, $M=8$

than $1 \mathrm{~dB}$ at $0.1 \%$ of CCDF. However, we get this performance with only $88 / 16384=0.54 \%$ of computational complexity by using the proposed algorithm. The complexity will be further reduced, if we use the simple adaptive technique which was proposed in (16).

To visualize the increase of complexity, we provide two figures, Figure 6 and Figure 7 . We use (17) and (18) to plot Figure 6 and Figure 7. As the number of subblocks or phase factors is increased, the complexity of ordinary PTS technique is increased dramatically. However, the complexity of the proposed EIF-PTS technique is not increased so dramatically when compared with the ordinary PTS technique.

\subsection{Power Spectral Density Analysis}

In this subsection, we present Power Spectral Density (PSD) analysis of the proposed algorithm. To show spectral leakage, we combine an ideal Pre-Distorter (PD) with High Power 


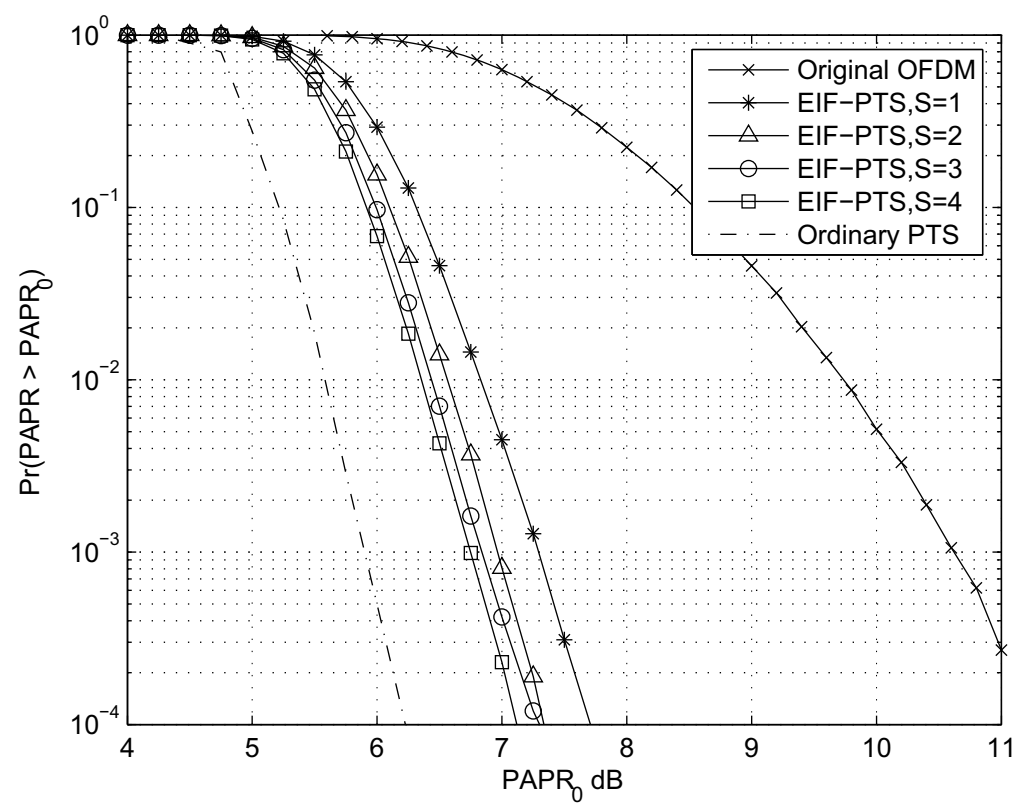

Fig. 5. Performance of EIF-PTS, $M=8$

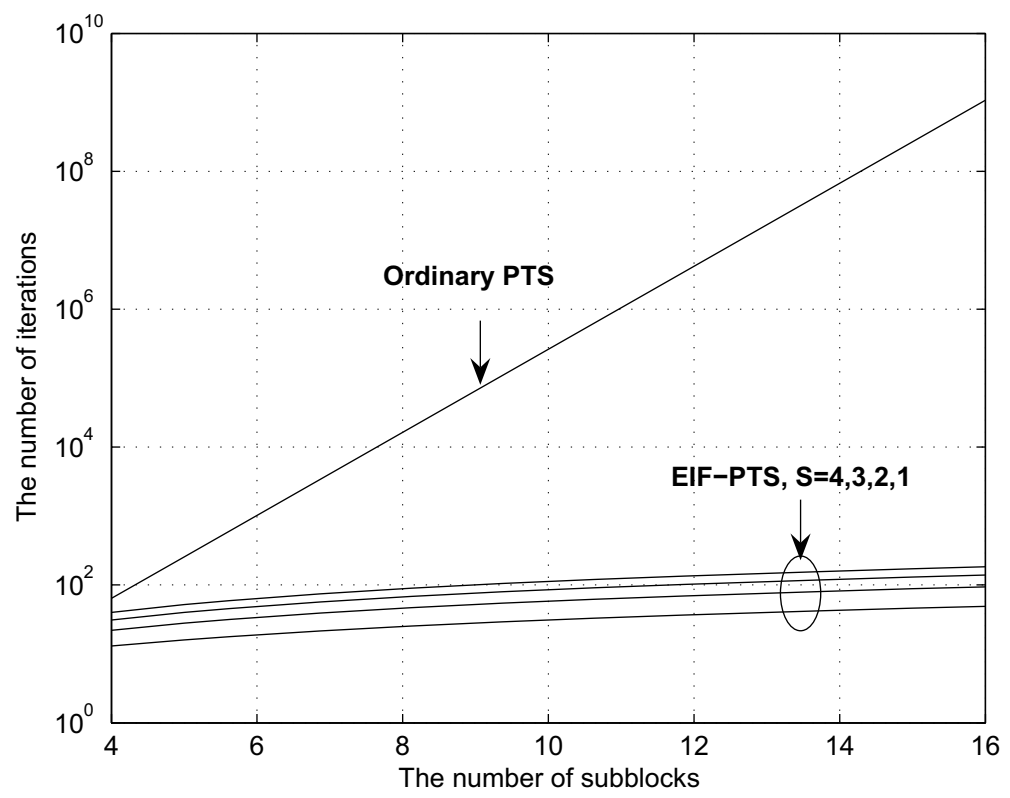

Fig. 6. Comparison of complexities between ordinary PTS and proposed EIT-PTS, $W=4$ 


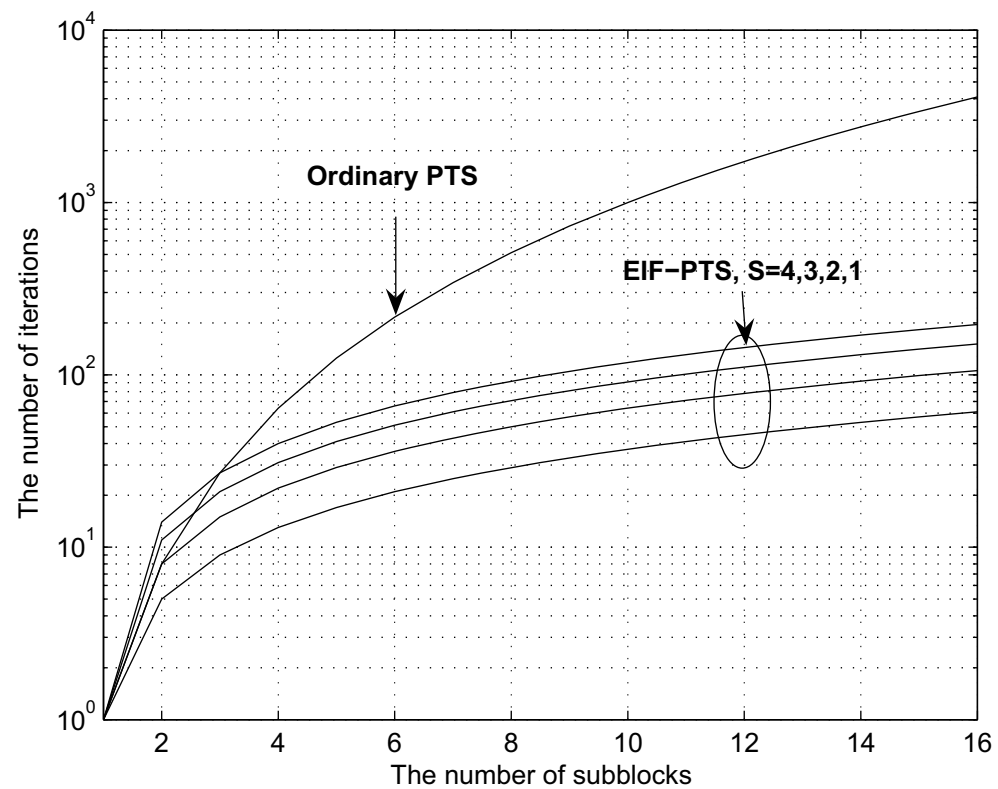

Fig. 7. Comparison of complexities between ordinary PTS and proposed EIT-PTS, $M=4$

Amplifier (HPA), as we did in (1). Thus the amplitude transfer function becomes a Soft Envelope Limiter (Please refer (1)). That is, the transfer function is linear up to a certain range and beyond that range, the signal is clipped. The spectral leakage is due to this clipping process. In Figure 8 , we use $M=4$ subblocks and set Input Back-Off $(\mathrm{IBO})=8 \mathrm{~dB}$. As we can see, without performing any PAPR reduction technique, even though we use an ideal PD and set high IBO which reduces power efficiency, we cannot avoid large spectral leakage. The proposed EIF-PTS algorithm can significantly reduce the spectral leakage and moreover it can also adjust the performance by adjusting the parameter, $S$. If we increase the number of subblocks from $M=4$ to $M=8$, we can get better performance even though we reduce the IBO from 8 to 7 (Figure 9).

\section{Conclusion}

One of the major problems associated with OFDM is its high PAPR. In this chapter, we proposed an enhanced version of the iterative flipping algorithm to efficiently reduce the PAPR of OFDM signal. There is an adjustable parameter so that one can choose based on performance/complexity trade-off. Simulation results show that this new technique gives good performance with significantly lower complexity compared with the ordinary PTS technique. 


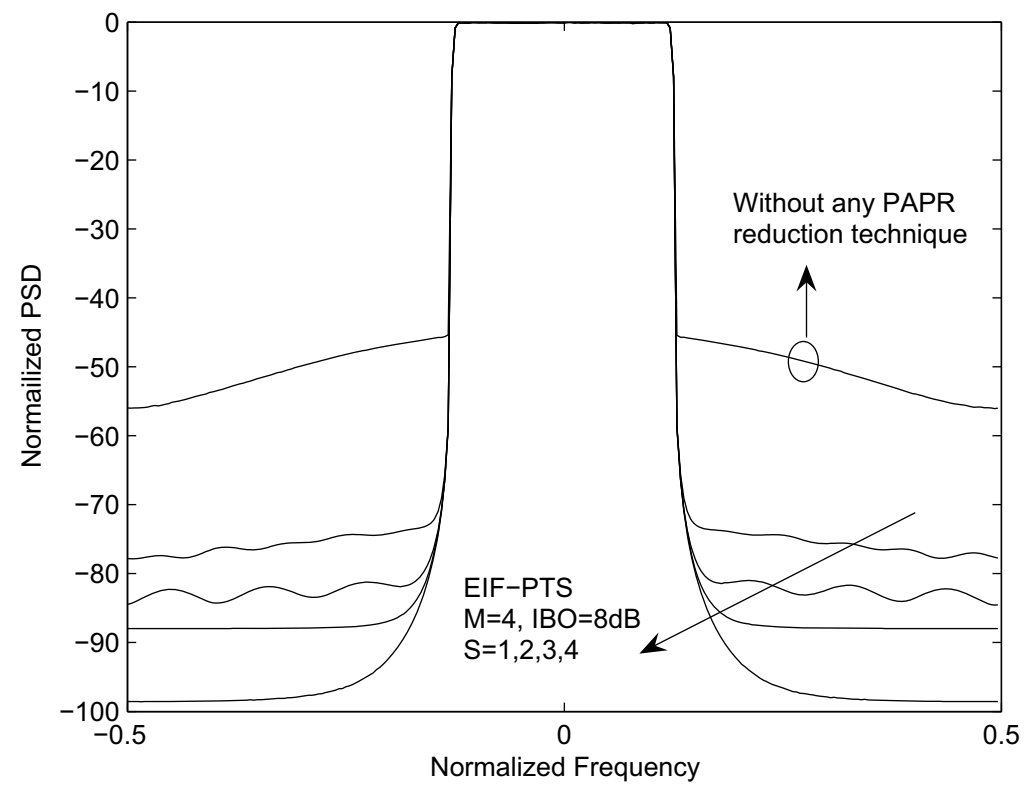

Fig. 8. Power Spectral Density of the EIT-PTS technique, when $I B O=8 d B, M=4$

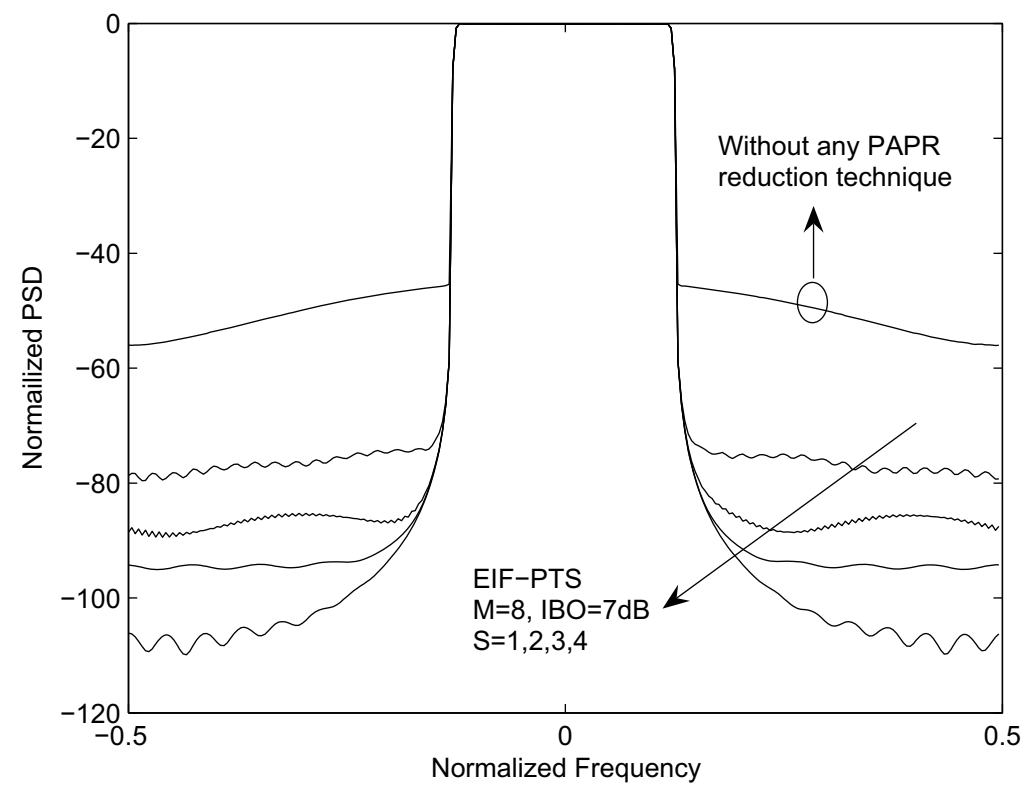

Fig. 9. Power Spectral Density of the EIT-PTS technique, when $I B O=7 d B, M=8$ 


\section{References}

[1] Byung Moo Lee and Rui J.P. de Figueiredo, "Adaptive Pre-Distorters for Linearization of High Power Amplifiers in OFDM Wireless Communications," Circuits, Systems $\mathcal{E}$ Signal Processing, Birkhauser Boston, vol. 25, no. 1, 2006, pp. 59-80.

[2] Byung Moo Lee and Rui J.P. de Figueiredo, "A Tunable Pre-Distorter for Linearization of Solid State Power Amplifier in Mobile Wireless OFDM," IEEE 7th Emerging Technologies Workshop, pp. 84-87, St.Petersburg, Russia, June 23 - 24, 2005.

[3] Rui J. P. de Figueiredo and Byung Moo Lee, "A New Pre-Distortion Approach to TWTA Compensation for Wireless OFDM Systems," 2nd. IEEE International Conference on Circuits and Systems for Communications, ICCSC-2004, Moscow, Russia, No. 130, June 30 - July 2, 2004. (Invited Plenary Lecture).

[4] Y. Kou, W. Lu and A. Antoniou, “New Peak-to-Average Power-Ratio Reduction Algorithms for Multicarrier Communications," IEEE Transactions on Circuits and Systems I, Vol. 51, No. 9, September 2004, pp. 1790-1800.

[5] X. Li and L. J. Cimini, "Effect of Clipping and Filtering on the performance of OFDM," IEEE Communication Letters, Vol. 2 No. 5, May 1998, pp.131-133.

[6] A.E.Jones, T.A.Wilkinson, and S.K.Barton, "Block coding scheme for reduction of peak to mean envelope power ratio of multicarrier transmission scheme," Electronics Letters, vol 30, pp. 2098-2099, December 1994.

[7] J. Tellado and J. M. Cioffi, "Efficient algorithms for reducing PAR in multicarrier systems," IEEE International Symposium on Information Theory, Cambridge, MA, pp. 191, Aug. 1998.

[8] V. Tarokh and H. Jafarkhani, "On the computation and reduction of the peak-to-average power ratio in multicarrier communications," IEEE Trans. Commun., vol. 48, pp. 37-44, Jan. 2000.

[9] S. H. Muller and H. B. Huber, "OFDM with reduced peak-to-mean power ratio by optimum combination of partial transmit sequences," Electronics Letters, vol. 33, pp. 368-369, Feb. 1997.

[10] L. J. Cimini, Jr. and N.R. Sollenberger, "Peak-to-average power ratio reduction of an OFDM signal using partial transmit sequences," IEEE Communication Letters, vol. 4, pp. 86-88, Mar. 2000.

[11] R. O'Neil and L. N. Lopes, “Envelop variations and spectral splatter in clipped multicarrier signals," in Proc. of PIMRC'95, Sept. 1995 pp. 71-75.

[12] L. Wang and C. Tellambura, "A Simplified Clipping and Filtering Technique for PAR Reduction in OFDM Systems," IEEE Signal Processing Letters, Vol. 12, No. 6, June 2005, pp. 453-456.

[13] J. Tellado, "Peak to average power reduction for multicarrier modulation," Ph.D dissertation, Stanford university, Sept. 1999.

[14] S. H. Muller and J. B. Huber "A Comparison of Peak Power Reduction Schemes for OFDM," IEEE GLOBECOM'97, Phoenix, Arizona, pp. 1-5, Nov. 1997.

[15] S. H. Han and J. H. Lee "PAPR reduction of OFDM signals using reduced complexity PTS technique," IEEE Signal Processing Letters, Vol. 11, No. 11, Nov. 2004.

[16] A. D. S. Jayalath and C. Tellambura "Adaptive PTS approach for reduction of peak-toaverage power ratio of OFDM signal," Electronics Letters, pp. 1226-1228, vol. 36, no. 14 6th July 2000. 


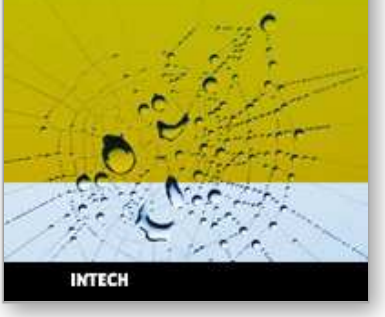

\section{Mobile and Wireless Communications Physical Layer Development and Implementatiom}

Edited by Salma Ait Fares and Fumiyuki Adachi

ISBN 978-953-307-043-8

Hard cover, 290 pages

Publisher InTech

Published online 01, January, 2010

Published in print edition January, 2010

Mobile and Wireless Communications have been one of the major revolutions of the late twentieth century. We are witnessing a very fast growth in these technologies where mobile and wireless communications have become so ubiquitous in our society and indispensable for our daily lives. The relentless demand for higher data rates with better quality of services to comply with state-of-the art applications has revolutionized the wireless communication field and led to the emergence of new technologies such as Bluetooth, WiFi, Wimax, Ultra wideband, OFDMA. Moreover, the market tendency confirms that this revolution is not ready to stop in the foreseen future. Mobile and wireless communications applications cover diverse areas including entertainment, industrialist, biomedical, medicine, safety and security, and others, which definitely are improving our daily life. Wireless communication network is a multidisciplinary field addressing different aspects raging from theoretical analysis, system architecture design, and hardware and software implementations. While different new applications are requiring higher data rates and better quality of service and prolonging the mobile battery life, new development and advanced research studies and systems and circuits designs are necessary to keep pace with the market requirements. This book covers the most advanced research and development topics in mobile and wireless communication networks. It is divided into two parts with a total of thirty-four stand-alone chapters covering various areas of wireless communications of special topics including: physical layer and network layer, access methods and scheduling, techniques and technologies, antenna and amplifier design, integrated circuit design, applications and systems. These chapters present advanced novel and cutting-edge results and development related to wireless communication offering the readers the opportunity to enrich their knowledge in specific topics as well as to explore the whole field of rapidly emerging mobile and wireless networks. We hope that this book will be useful for students, researchers and practitioners in their research studies.

\section{How to reference}

In order to correctly reference this scholarly work, feel free to copy and paste the following:

Byung Moo Lee and Rui J. P. de Figueiredo (2010). An Enhanced Iterative Flipping PTS Technique for PAPR Reduction of OFDM Signals, Mobile and Wireless Communications Physical Layer Development and Implementatiom, Salma Ait Fares and Fumiyuki Adachi (Ed.), ISBN: 978-953-307-043-8, InTech, Available from: http://www.intechopen.com/books/mobile-and-wireless-communications-physical-layer-developmentand-implementatiom/an-enhanced-iterative-flipping-pts-technique-for-papr-reduction-of-ofdm-signals

\section{INTECH}




\section{InTech Europe}

University Campus STeP Ri

Slavka Krautzeka 83/A

51000 Rijeka, Croatia

Phone: +385 (51) 770447

Fax: +385 (51) 686166

www.intechopen.com

\section{InTech China}

Unit 405, Office Block, Hotel Equatorial Shanghai

No.65, Yan An Road (West), Shanghai, 200040, China

中国上海市延安西路65号上海国际贵都大饭店办公楼 405 单元

Phone: +86-21-62489820

Fax: $+86-21-62489821$ 
(C) 2010 The Author(s). Licensee IntechOpen. This chapter is distributed under the terms of the Creative Commons Attribution-NonCommercial-ShareAlike-3.0 License, which permits use, distribution and reproduction for non-commercial purposes, provided the original is properly cited and derivative works building on this content are distributed under the same license. 